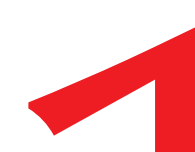

Abstract

Across Canada, the rates of many sexually transmitted infections (STI), including HIV, continue to fluctuate, with the numbers of new infections increasing within many subpopulations. This warrants an evaluation of the Public Health Agency of Canada's clinical guidelines for STI management. To accomplish this, Gagnon and Simon's work on sexual scripts has been used to explore the structure of current STI clinical practice. This theoretical analysis reveals some of the shortcomings of PHAC's guidelines, and identifies how they reduce patients to vectors of illness and disease.

Key Words assessment, HIV, nursing, sexual health, sexual script theory, sexually transmitted infections

\section{Theory and Practice:}

\section{Examining PHAC's STI Assessment Guidelines Using Sexual Script Theory}

\section{PATRICK O'BYRNE \& JESSICA WATTS}

\section{Introduction}

In light of recent research which indicates that front-line, often nursing-based, sexually transmitted infection (STI) and HIV clinical practice rarely has any effect on STI/HIV transmission (for example, in Canada, the rates of many STIs/HIV have increased over the last several years, despite an increase in resource expenditure in this field), a thorough evaluation of the Public Health Agency of Canada's (PHAC) clinical guidelines for evaluating a patient's need for STI/HIV testing is warranted.[1-7] Problematically, the current stateof-affairs in STI/HIV management is that while PHAC's STI/
HIV assessment guidelines arise from rigorously undertaken research, discussions about the meaning and significance of these findings within the clinical domain rarely occur. To initiate a discussion of this topic, this paper will present the findings of an analysis of the PHAC's national guidelines for the clinical management of STIs/HIV[8] that employed Gagnon and Simon's $[9,10]$ sexual script theory. An overview of this theory, what it reveals about the PHAC guidelines, and some clinical implications that arise from such an analysis are presented below.

\section{A theoretical framework about human sexuality: sexual script theory}

In contemporary society, most conceptualizations of human sexuality are based on biological (evolutionary) theories of species propagation and psychoanalytic ideations about desire and pleasure.[11-13] While these approaches are often accepted without critique, some authors have voiced their opposition. Simon and Gagnon, [9] as one example, describe sexuality as a learned behaviour that is embedded 
in a complex set of social scripts which are specific to particular socio-political cultures and histories. Their analyses of sexuality suggest that, while "what we conventionally describe as sexual behaviour is rooted in biological capacities and processes, ...the sexual area may be precisely that realm wherein the superordinate position of the sociocultural over the biological level is most complete".[9 p15] Here, Gagnon and Simon[9] clearly identify that one can deny neither the biological, nor the social aspects of human sexuality. Instead, sexuality should be understood as a scripted practice which arises as a result of numerous interactions between an array of sociocultural factors and biological conditions.

Other authors who share Gagnon and Simon's rejection of conventional biologically-based ideations about human sexual behaviour are Judith Butler and Erving Goffman. Indeed, similar to Gagnon and Simons' dramatic metaphor is Butler's work on performativity and Goffman's ideas about the performance of self in everyday life. In both of these cases, of which the latter preceded Gagnon and Simon's work, these two authors argue that human behaviour is a performed activity, a script to be followed one could say. What is important about Goffman and Butler's work in this context is that it clearly articulates how performativity should be interpreted: it does not signify that behaviour is always consciously intentionally acted, but rather, that sexual behaviours are not innate or natural.[14,15] They are not expressions of underlying biological predispositions, that is. Performativity and the performative nature of human behaviour, as Butler and Goffman conceptualize it, thus suggests that human behaviour is learned and executed within specific contexts. It is also within these contexts that this behaviour is interpreted and understood. For Butler specifically, this means that gender cannot be thought of as a role that portrays an inner self; it is an act which portrays the social falseness of the psychological interior that is publicly regulated in social situations.[14] Gender cannot be real or fake from this perspective. This aligns with both Goffmans' work and Gagnon and Simon's ideas, wherein, first, the individual's identity is constructed based on the context and, second, roles are exchanged depending on the social situation.[15] Where Gagnon and Simon's work excels in relation to these other two authors, however, is in their clear and simple use of the dramatic metaphor.

\section{The dramatic metaphor}

Gagnon and Simon[16] use a dramatic metaphor to explain the complexity of human sexual activities (performance), and their work in this situation outlines a clear structure for understanding the social nature of sexual behaviour. To explain further, Gagnon and Simon construct a metaphor, which gives script theory its name, and it comprises the following components - Act (the activity), Scene (the context of the activity), Actor (the one who performed the act), Performance (how it was done), and Motivation (why it was done). It also seeks to emphasize that sexual encounters are multilayered performances that include the actual sexual practices undertaken, the people and places involved, and the reasons for which the performance was enacted. The purpose of this metaphor is to provide an easily understood framework for discussing human sexuality. As an example of this process, the act could be oral, anal, or vaginal sex; the scene could be a swinger party, circuit part, or a quiet room; the actors could be a male-female, male-male, femalefemale, trans-trans couple, or any other combination thereof; the performance might include which individuals performed which sexual roles; and motivation would encompass the purpose of the sexual encounter - pleasure, conception, rule breaking, fantasy, etc. As can be seen in this example, Gagnon and Simons'[165] dramatic metaphor can be satisfactorily used to guide in-depth analyses of human sexuality in ways that align with Butler and Goffman's ideas that behaviour is performative.

\section{What are sexual scripts?}

What is particularly beneficial about Gagnon and Simon's work (particularly in comparison to other writings that conceptualize human behaviour as performative, such as, Butler, Goffman, and Foucault), is that Gagnon and Simon[9] use sexual scripts to explain in a very practical manner why some situations culminate in sexual activity, while others do not. This, Gagnon and Simon argue is the case because sexual scripts "are involved in learning the meaning of internal states, organizing the sequences of specifically sexual acts, decoding novel situations, setting the limits on sexual responses and linking meanings from non-sexual aspects of life to specifically sexual experience". [9 p17] In other words, sexual scripts are cognitive schemata that individuals (who can be considered actors) use to organize and give meaning to a variety of otherwise ambiguous situations. These scripts thus determine whether social interactions (performances, that is) have the potential to yield sexual outcomes.

The term script is thus appropriate because, for a situation to become (or be perceived as) sexual, a series of sequenced events must occur in the proper order (the script), in the correct place (the stage), and involve the appropriate people (the actors). In fact, the environment in which a social 
interaction takes place often influences if it will become a sexual encounter - for example, the interpretation of mouthto-mouth, or mouth-to-skin contact will vary depending on whether it occurs in sexualized environments (e.g., bathhouses, swingers clubs, circuit parties), or in nonsexualized, settings. Thus, for Gagnon and Simon, place is an important attribute in any sexual encounter because individuals must ascribe a sexual nature to a milieu for it to play a role in culminating a sexual situation.

Moreover, when at least two people encounter one another in a place that they evaluate as an acceptable sexual locale, a requisite series of preceding factors (a designated set of indicators) must occur, and be recognized, to produce a communal interpretation that a given event has the potential to become sexual. Furthermore, the actors involved must interpret these signals both as sexual and desirable: that is, in a given situation, everyone's interpretation must correspond - all must perceive the situation as sexual and each actor must be able and willing to properly continue the sequence (the script) to ensure a sexual outcome. If this script is not followed, Gagnon and Simon[9] propose that the likelihood of a consensual sexual interaction greatly diminishes.

For the above reasons, Gagnon[17] argues that sexual conduct is a product of context and internal negotiation, rather than an instinctive drive as interpreted by Freudian tradition. That is, sexual script theory maintains that sexuality is internally negotiated, and based on social norms; it is a force that is partially under individual control and regulation, and partially the outcome of social dictates. For example, while sexual contact is internally negotiated before it is undertaken (excluding situations of sexual assault), beliefs about what constitutes normal sex are based on social values and assumptions. It is the social construction of a subjective reality, Foucault might argue. $[18,19]$ This signals that people do not become sexually active without thinking about it, internally negotiating the act, and applying the correct script within the pre-established parameters and context of their culture and history. This category of thinking, however, should not be interpreted as a rational, cognitive process. Instead, this process is a visceral level analysis that evaluates if social dictates correspond with personal feelings about a given sexual practice, an assessment made within the individual's own mind, wherein s/he acts as a fantasist by taking cultural norms and modifying them to form new ways of looking at cultural scenarios and interactions.[17)] Thus this aspect of Gagnon and Simon's[16,20] work explains much of the individually based variation that occurs in relation to prevailing socio-cultural sexual norms.

\section{The underlying principles of sex script theory}

With the foregoing understanding in hand, we will now review the fundamentals of sexual script theory. The theoretical assumptions are as follows:

1.Sexuality is not a constant or universal phenomenon that is independent of culture and historical period. Instead, it is expressed differently depending on the era and culture in which it occurs. Therefore, the social and cultural aspects that surround sexuality are not simply responses to biological and developmental sexual imperatives; they are the outcomes and outputs of culturally based ideations of normality, acceptability, and humanity.[17]

2.The meaning associated with sexual conduct differs across cultures and historical periods. Despite the fact that there are often only a limited number of sexual practices being undertaken, the meanings associated with these activities vary greatly. As a result, cross-cultural comparisons must always be approached with caution. [17] One cannot assume that just because the sexual practices of a culture are similar to those within another culture, the meanings embedded within these sexual practices are identical. Thus, one must be cautious when either comparing or contrasting the sexual practices of different cultures and different time periods particularly if the goal is to establish or determine which factors differ or remain constant between different groups.

3.Science, including its techniques of data collection and paradigms of inquiry, is a product of the culture and historical period in which it exists. Therefore, it is impossible for science to be completely objective.[17] In relation to human sexuality, scientific inquiry is a mechanism, which often does little more than replicate already established ideations about human sexuality. In other words, science simply identifies different aspects of sexual scripts, while remaining a component of these scripts, and without challenging their basic assumptions.

4.Individuals learn how to be sexual, including the movements that define sexual expression, within the confines, rules, and regulations of a specific culture. Every aspect of one's sexuality, from arousal to pleasure to climax, is, therefore, a learned process.[21,22]

5.In most societies, both sexual and gender conduct, are, to some degree, linked. The result is that men and women may express their sexualities differently - including associating different experiences and emotions with sexual conduct. However, these differences are often referred to as biological in origin - Sigmund Freud. Gagnon[17] cautions 
that examining gender differences as "natural differences" in relation to sexual conduct is erroneous.[17] Gender and sexuality are both learned social practices, and as such, cannot be understood without an appreciation of the culture within which they occur. $[23,24]$

\section{Levels of sexual scripts}

Building on the above assumptions, Gagnon and Simon $[16,20]$ argue that there are three components to sexual scripts (cultural, interpersonal, and intrapsychic), and that it is the interaction of these levels that creates specific sexual encounters in different circumstances.

\section{The cultural level}

The cultural component of sexual script theory comprises highly abstract, group level sexual norms. Here, scripts are elementary "instruction manuals" that provide rough guidelines for what constitutes appropriate sexual behaviour within groups.[9] Sexual scripts are thus impersonal, and do not describe the actions or beliefs of any specific individual.[9,10] Instead, cultural level scripts outline the general properties of different groups' sexual standards, such as, what constitutes generally acceptable dating practices, or more specifically, who may penetrate which orifices when and how. The outcome of these sexual scripts is that cultures can be identified based on them[25] and such identification helps the members of these groups to interpret the actions and practices of the people whom they consider as group members.

However, the cultural level aspects of sexual scripts are not formally codified. Rather, cultural sexual scripts are often transmitted orally from person-to-person during conversations with intimates. Therefore, an outsider might experience difficulty in identifying the sexual scripts of a given culture because they are usually not publicly discussed. Nevertheless, over the last few decades, key pieces of research have been developed, which overview the cultural norms of diverse sexual groups; for example, Humphreys'[26] exploration of the Tearoom Trade, or Holmes' and colleagues[27-30] investigations of gay men who attend bathhouses.

\section{The interpersonal level}

At the interpersonal level, Gagnon and Simon[16,20] posit that abstract cultural rules affect person-to-person dealings as a result of self-observation. These authors propose that individuals ensure that they behave according to the dictates of their cultural sex scripts by scrutinizing their own behaviour, and observing and evaluating themselves in the same way that they watch and appraise others. It is an active "I" examination of the passive "me", which occurs at the level of social interaction. Goffman[31] wrote The Presentation of Self in Everyday Life, describing how individuals self-monitor their behaviour to ensure that it corresponds with what they feel is context appropriate. From a Foucauldian[18,19] perspective, this is the ethics of self that occurs within an overarching cultural environment.

Regardless of specific theoretical perspective, however, it is safe to say that in a dyadic interaction there are two evaluators scrutinizing the behaviour of each other, with each using that which they deem appropriate sexual behaviour as the benchmark for evaluating both themselves and the other. This signifies that people often allow their conduct to be determined by the expectations of their social group, and will modify their behaviour if they feel that their sexual practices are dissimilar to the sexual conduct and sexual expectations of other group members.[16,20] For example, if an individual perceives that the majority (or an influential minority) of his/her social group engages in a specific form of sexual activity (e.g., unprotected oral sex), in many cases this individual will become more likely to engage in the same type of sexual practices to ensure compliance with group normative behaviour. $[9,10,32]$

\section{The intrapsychic level}

While Gagnon and Simon[16,20] argue that individuals structure their behaviour according to cultural norms, they do not suggest that people are unable to act autonomously. Instead, they suggest that, despite the influence of social norms, people remain capable of intrapsychically evaluating potential actions before engaging in them. Thus, when people evaluate cultural mores, they produce individualized sexual scripts that closely, but not exactly, resemble cultural sexual norms. As part of this interaction between individual thoughts and overarching cultural norms, each person performs the roles of audience, critic, and reviser of cultural scenarios; thus, individuals internalize cultural level sex scripts, analyze them according to personal beliefs and perceptions, and then change these scripts to make them congruent with personal beliefs.[17] This third level is often non-existent in other theories. Gagnon and Simon recognize the importance of the individual, and consequently include it within their sexual script theory.

The outcome of this inclusion is that Gagnon and Simon insist that individuals simultaneously act out sexual scripts according to social standards and modify them in relation 
to their personal values. This can occur both before and after an actual sexual encounter. For example, individuals may idealize a sexual experience when reflecting back on it, transforming it into a romantic, steamy, or well-executed experience. Unfortunate aspects of this experience, such as pain, clumsiness, alcohol consumption, or personal deviations from cultural norms may be deleted from memory and idealized feelings of closeness and love, raw sexuality and pleasure, or personal skill and sexual proficiency may be all that remain. This continual editing process explains how, despite the existence of cultural norms, individuals have varying preferences for, and perceptions of, sexual contact.

Another aspect of sexual scripts at the intrapsychic level is their wide range of completion - from brief memories or fragments of desires to well ordered and completely outlined plans. In fact, the closer an individual moves towards putting a script into action, the more complete the script becomes. Thus, the most complete scripts are those that individuals intend/desire to enact; time and energy has already been invested in their conceptualization. When individuals engage in this precise mental scripting process, they perform what Gagnon and Simon[16,20] call the role of playwright. As part of this, individuals interpret their cultural-level scripts based on their personal ideologies, produce pre-scripted ideas about how they would like specific sexual encounters to unfold, and attempt to perform their sexual activities in accordance with their personally-modified, culturally-based scripts. $[16,20]$ It is important to remember, however, that the actual execution of these scripts is always modified by interpersonal interaction.

\section{Interaction of the three levels of script}

While, so far, the three levels of sexual script theory have been presented as discrete, they are actually intricately intertwined. Through the interpersonal and intrapsychic levels of scripting, individuals form narratives that compose conduct into a series of events. If these events occur on a regular basis, individuals become better able to recognize them, and draw on the appropriate script when they occur. Theoretically, this means that the more experience one has, the more one should be able to navigate sexual scripts successfully and skilfully. Furthermore, from cultural level scripting, the individual acquires a set of narratives, which establish the degrees of appropriateness of given sexual practices. If the cultural values change, then the set of instructions for conduct also change, including the instructions for good and bad sexual conduct. It is important to remember that from the social constructivist perspective within which this theoretical approach is situated, all social behaviour, including sexual practices, is created. Consequently, sexuality is more than merely individual behaviour; it is a reflection of culture.[17]

\section{Evaluating the PHAC guidelines using sexual script theory}

As part of a sexual health assessment, health care professionals typically ask their patients a series of questions. As directed by the PHAC STI/HIV assessment guidelines, [8] these questions can be grouped into six main categories: relationship status, sexual risk behaviour, STI history, reproductive health history, substance use history, and psychosocial history. In this section, sexual script theory will be used to examine the first three of these assessment categories: relationship status, sexual risk behaviour, and STI history.

\section{Category 1: relationship status}

In the first category of the PHAC[8] guidelines, clinicians are instructed to collect information about the status of their patients' relationship(s). This includes questions about regular sexual partners, the duration of this/these relationship(s), and if any concerns exist regarding this/these relationship(s) (e.g., violence, concurrent partnerships, etc.). The assumption underpinning these questions seems to be that individuals who are in stable, long-term relationships are less likely to acquire either an STI or HIV.

However, regardless of the real-life association between these behavioural factors and actual STI/HIV acquisition outcomes, when these questions are examined in greater detail, and are approached from the perspective of sexual script theory, a few major problems arise. Most significantly, this occurs because the PHAC[8] guidelines fail to acknowledge that an individual's interpretation of what constitutes normal and acceptable sexual activity, and thus what amounts to a relationship, is dependant on an individual's cultural and historical context. Sexual script theory informs us that clinicians must be aware (although the PHAC provides no guidance on this matter) that the term relationship should not be indiscriminately employed without prior clarification of what it means to the individual patient because the predictive factors for STI/HIV acquisition are dependent on the complexities which are inherent in asking questions and gathering information about this term.

Gorbach and Holmes[33,34] emphasize this point based on research, which has shown that, depending on an individual's (intrapsychic) interpretation of culturally defined relationship norms, his/her likelihood for STI/HIV acquisition varies 
within the same categories of regular/casual and long/shortterm relationships. This variance occurs because different social groups have (1) varying cultural norms about condom use with regular versus non-regular partners, (2) assorted group level acceptability about having sex with non-regular partners while in a long-term relationship, (3) diverse social standards regarding women's power to negotiate safer sex with regular versus non-regular partners, and (4) differing personal beliefs about the nature and meaning of one's personal relationship(s). To explain this last point further: research in STI clinics has found a great deal of discordance between how different partners in the same relationships describe their relationship status; for example, while one partner may feel that the relationship is monogamous and stable, the other may have described it as casual.[35] The outcome is that, when these factors fluctuate, the information that is gathered about a patient's relationship status may be of little use to STI/HIV clinicians if it does not address the cultural level sexual scripts which inform a given patient's interpretation of what constitutes a relationship and what occurs within its confines.

Of concern here is that the PHAC[8] guidelines seem to assume that being in a stable, long-term relationship is beneficial from an STI/HIV perspective, which is true if neither of the two partners has any previous infections, and if neither is having sex with others during the period of their relationship. Unfortunately, this assumption overlooks the research which suggests that cultural norms for many women foster false beliefs about their male partners' proclivity/ opportunities for engaging in sexual relations with other partners, and thus renders them considerably more likely to acquire an STI/HIV.[36] Other research also indicates that the cultural norms in open relationships among some gay men, wherein specific rules for protected or non-penetrative sex exist for non-regular partners, actually enhance the likelihood of STI/HIV acquisition because only a few individuals within these relationships seem to consistently maintain the rules of safer sex with non-regular partners. $[37,38]$ In these cases, stable relationships permit sex with others, but not always in the way that each partner assumes.

Moreover, the first category in the PHAC guidelines also instructs clinicians to ask their patients if they have any concerns about their relationship(s), including violence, abuse, or coercion. While this is an area of great importance (because the patient may be in a situation of violence that has never been addressed), the PHAC guidelines fail to take into consideration the cultural implications of this question. Here, a distinction must be made between what constitutes violence and what amounts to abuse. For example, in the context of sadomasochism, what might be considered violent or abusing by some may be considered pleasurable by others. It is also important to remember that, within most societies, gender and sexuality are linked to some degree, and that this results in men and women learning to equate different emotions and experiences with differing aspects of human sexuality. This process can ultimately lead to some women normalizing certain associations that, from the perspective of other cultures, may seem horrific; female genital mutilation is one such example.

Therefore, Gagnon and Simon's[9] sexual script theory reveals that if clinicians inquire about their patients' relationships, but do so without any theoretical understanding of how this social construct can vary based on culture, time, age, and so forth, they may accidentally ignore the cultural and historical implications imbedded in the term relationship. In doing so, a clinician might also fail to acquire a sophisticated and nuanced understanding of the complex interactions between a patient's relationship status (how ever s/he defines it) and his/her probability of acquiring an STI or HIV.

\section{Category 2: sexual risk behaviour}

In the second category of the PHAC guidelines, clinicians are instructed to collect information regarding their patients' sexual behaviour. This includes questions about: (1) the number of different sexual partners, (2) sexual preference/ orientation, (3) sexual activities undertaken, and (4) personal risk evaluation. As in the previous section, these questions provide a fairly comprehensive assessment of the individual's sexual practices, but still fail to incorporate any information that goes beyond the individual (intrapsychic) level. These guidelines are thus incomplete because they do not adequately guide clinicians regarding what to ask in order to undertake counselling. While one might argue that being aware, for example, that the patient engages in unprotected anal sex with a number of different sexual partners is all that the clinician needs to know to construct effective counselling, research has indicated that this is not always the case.[39]

To explain further: when clinicians counsel their patients to avoid certain sexual practices for STI/HIV reasons, but do so without exploring the meaning and significance of these sexual acts for their patients, they may be providing advice that is incompatible with their patients' perceptions of sexuality. Counselling that is based on the clinician's opinion, rather than on the beliefs of the patient, will probably accomplish very little. A study by Holmes and Warner[40] supports this hypothesis. These two authors undertook an in-depth 
qualitative investigation of bareback sex, and uncovered that, for some men, the practice of unprotected anal sex is not only about enhanced sensation during penetration, but is also about the sensations of "desire, transgression, and pleasure" that are associated with the exchange of semen.[40 p10] Thus, if clinicians counsel patients such as these about methods to diminish STI/HIV transmission by advocating for increased condom use, they will be promoting a harm reduction strategy that fails to acknowledge the erotic aspects of their patients' sexual experiences, and therefore will likely be ignored.

This not only emphasizes that clinicians who work in sexual health must be aware of the socio-sexual cultural norms of their patients if they wish to provide meaningful counselling to their patients, but also indicates the need for further research addressing the socio-sexual norms of diverse groups because understanding each patient's sexual scripts would indicate why individuals behave the way they do, and would help to form the basis for person-specific sexual health counselling. $[16,20]$ In the field of STI/HIV care, such an approach could yield beneficial results.

As previously stated in the description of sexual script theory, across cultures and time periods, there is little difference in the motions of sexual activity, but the meaning associated with them varies greatly. Therefore, although questions about specific sexual health practices will succeed in identifying which type(s) of intercourse were undertaken, and may enable clinicians to recommend a matching STI/HIV testing method, this does not take into account the meaning of this activity for the individual, and therefore, misses a key point in a complete health assessment: knowing the meaning of a given sexual practice for the individual is an important part of an assessment because it enables clinicians to choose more specific/appropriate counselling. For example, in the case of barebacking, it might be more useful for clinicians to suggest an increased use of lubricant to decrease the possibility of epithelial tearing rather than to recommend condoms. Anal foreplay or the sexual practice of skeeting[41] might also prove to be relevant counselling options.

Although discovering why individuals engage in the sexual practices that they do is clearly not the goal of the PHAC guidelines, perhaps this is a shortcoming regarding their effectiveness. Specifically, the PHAC guidelines may not be adequately directing STI/HIV clinical practice because they focus almost exclusively on reducing patients to a matrix of risk factors; this reduces complex human beings to metaphorical "petri dishes", each with varying degrees of probability for becoming the host of an array of sexually transmissible pathogens. This process (at least as it is presented in the PHAC STI/HIV guidelines), seems to occur without any appreciation of how the information can be used to structure individual counselling. Indeed, another section of this manual recommends counselling patients to always use condoms; the manual reads: "if practicing anal intercourse, always use a condom and extra lubrication, and avoid use of spermicidal condoms".[8 p17] Harm reduction based counselling is alluded to, but seems to be based on clinician-directed strategies, not on those which might be feasible for, and desirable to, patients.

Consequently, questions that aim to address only the "who", "what", "where", and "when" of sexual behaviour lend themselves to quantitative answers, but ignore qualitative explanations. One may understand a patient's need for protective barriers, but will not have any understanding of why this protection is, or is not used. The use of sexual script theory as a theoretical guide for this assessment process could, however, yield details about why a given patient had sexual relations without a condom with an individual who was not their regular partner. In this way, the clinician who is performing the interview might acquire more knowledge about how s/he might best intervene to decrease the likelihood of his/her patient acquiring an STI or HIV because, by asking these questions, clinicians can better understand their patients' motivations, expectations, and desires. Such a recommendation could be implemented by having clinicians ask questions about the cultural, interpersonal, and intrapsychic aspects of their patients' sexual scripts, and then applying research that investigates specific sexual sub-cultures in their practice. However, in saying this, it is important to recognize that the added benefits that could arise from additional sexual history-taking must be balanced against the potential harm of patients feeling stigmatized by this line of questioning. This is an area in need of further exploration.

Other problems also arise when the content of the PHAC assessment guidelines are examined in more detail. For example, the PHAC assessment question about sexual orientation is problematic, most notably, because it is exclusively quantitative in nature, and completely ignores the premise of Gagnon and Simon's[9] sexual script theory that human sexuality is dependant on both culture and time. By restricting sexual orientation to the options of men, women, or both, this question normalizes a contemporary social idea that these choices constitute a mutually exclusive and exhaustive list of possibilities, and assumes that these options are immutable manifestations which arise from 
nature. Because this approach disregards the idea that human biology exists as anything other than the two distinct poles of male and female, it overlooks the possibility that patients have had sex with transgender, transsexual, and/or intersex individuals. It also disregards the fact that patients may be transgender, transsexual, or intersex themselves. Thus, the PHAC guidelines enforce a specific and restricted cultural conceptualization of sexuality - i.e., that the human body can be only one of two phenotypes. Here, the PHAC guidelines serve, not only as an assessment tool, but also as a method by which dominant cultural conceptualizations of sex, gender, and sexuality may be imposed on individual patients whose socio-sexual identity may be different.

Another question of concern in the PHAC guidelines deals with the ways in which patients meet their sexual partners. This is useful in situating a patient within given sexual networks (which may have higher or lower incidence/prevalence levels of specific STIs/HIV), but nonetheless fails to account for the socio-sexual cultural norms of these networks. Sexual script theory postulates that sexual behaviour is the result of cultural norms that individuals internalize and compare with interpersonal norms at the intrapsychic level. However, when the PHAC guidelines advise assessing the locations for meeting, arranging, and having sex that a patient chooses, they base these recommendations on epidemiological indicators (e.g., group and locale specific incidence and prevalence rates for STIS/HIV), and exclude any understanding of why people use some environments and not others to coordinate their sexual activities. By reducing places of sexual contact to little more than a criterion on a risk assessment matrix, clinicians are left without any in-depth knowledge that could be used to permit patients to engage in the sexual practices they desire in the milieu they desire, while minimizing their likelihood of STI/HIV acquisition/transmission. Sexual script theory highlights the importance of understanding such cultural norms and intrapsychic interpretations about sexuality, and as such, identifies a potentially major shortcoming of the current PHAC STI/HIV assessment guidelines: it is focused too precisely on specific behaviours to the absolute exclusion of any understanding about the cultural and personal significance of these practices.

The next question in this category of personal risk evaluation concerns the use of condoms and the factors that influence an individual to use, or not use protection. Culture can play a huge role in influencing the use of protective barriers: for example, while some cultures forbid condom use for religious reasons (e.g., strict Roman Catholics), others forego condoms based on beliefs that these objects constitute signs of distrust.[42] It is important to note that the question of "what influences your choice to use protection or not?", as scripted by the PHAC guidelines, seems to be the first time that this publication manifests cultural sensitivity. By allowing individuals to identify why they choose to use, or not use, protection, the clinician is better situated to select interventions that are appropriate for their patients. For example, at the intrapsychic level, they might explore their patients' perceptions and beliefs about condoms and other protective measures, or provide educational material on the benefits of protective barriers in an effort to change the patient's opinion about this subject.

However, clinicians must be careful when promoting condom use at the individual level because the use of protective barriers during sex typically occurs in dyads, and has to be negotiated between sexual partners[43] - a practice that sexual script theory identifies as occurring on an interpersonal level.[9] As such, an important question might be whether patients feel comfortable broaching the topic of condom (and other barrier) use with their partner(s). If not, methods by which discussions about protection could be initiated - an ideal topic for counselling. This stresses the idea that the PHAC recommendation that condoms are beneficial is not sufficient for real-life condom use, particularly if such intrapsychic interpretations conflict with the patients' cultural norms, or if individuals are unable to enforce their intrapsychic beliefs during an interpersonal interaction.

As the final question within this section of the PHAC guidelines, clinicians are instructed to ask their patients to identify what they feel is their own personal risk quotient for $\mathrm{STI} / \mathrm{HIV}$ acquisition - ranging from no risk to high risk. While it can be argued that the outcome of this question could generate meaningful information for counselling, the PHAC guidelines fail to acknowledge that patients' intrapsychic interpretations will influence their perceptions of relative risk. Undoubtedly, this intrapsychic assessment will involve a personalized interpretation of how mainstream scientific discourses and cultural norms intersect regarding STI/HIV information. Clinicians must then evaluate how accurate they feel that their patients' assessment is, based on the information that the patient has already supplied.[8] Problematically, this exercise can easily become an examination of one sexual culture's beliefs by another (and perhaps more dominant) culture. For example, even if a patient's sexual behaviours are considered high risk by a clinician, the patient may rate him/herself as low risk due to the perceived cultural norms and the relative activities of his peers.

Here, the PHAC guidelines attempt to objectify risk, and in 
doing so, strip this concept of its highly subjective content and inject it with political influence.[44-6] An assessment of a patient's perception of risk is thus transformed from being a personal evaluation into an examination of self that determines if a patient's behaviour corresponds with hegemonic ideals about sexual expression. [30] From the perspective of clinical sexual health practice, wherein the therapeutic relationship is of the impost importance in ensuring (1) that patients do not feel stigmatized, (2) that they feel comfortable asking potentially embarrassing questions, and (3) that they openly express a variety of intimate and personal information, this top-down appraisal of a patients' behaviour may not facilitate the overall goal of clinical sexual health practice: to reduce both the individual and the population level negative sequalae associated with STIs and HIV. $[47,48]$

\section{Category 3: STI history}

The third and final category of the PHAC STI/HIV assessment guidelines to be discussed here instructs clinicians to (a) gather information about their patients' previous STI screening practices, including past STI/HIV diagnosis, (b) evaluate if patients have had any sexual contacts of concern, and (c) determine if patients are experiencing any STI/ HIV-related symptoms. The purpose of these questions is multipart: previous diagnosis with an STI renders one more likely to acquire a second STI within the near future; sexual contact with a partner who is known to have an STI/HIV considerably increases one's probability of infection, and the existence of STI/HIV-related symptoms can impel empiric treatment. $[49,50]$ Based on this, these questions seem both simple and highly relevant.

However, sexual script theory identifies that the PHAC guidelines fail to recognize that human sexuality is the culmination of many cultural and interpersonal aspects there is absolutely no recognition of the cultural aspects of human sexuality in this section of the assessment guidelines. This could lead to problems because in some cultures STIs and HIV/AIDS are considered so deeply shameful that they are kept secret.[51-53] Consequently, questions about STI/ HIV history might, on one hand, exacerbate the feelings of shame and/or guilt that reduce STI/HIV health-seeking, and on the other hand, yield clinically useless (invalid) information. Such is the case when a patient with a history of gonorrhoea fails to disclose this information because of cultural perceptions of uncleanliness or shame as a result of having had this diagnosis.

Cultural awareness about the nature and meaning of specific sexual practices should also inform clinicians regarding their techniques of data collection. As Goffman[31] revealed, many individuals will present themselves in the social arena in ways that seem to fit the situation, or which appear to be desirable, regardless of the accuracy of this presentation - this phenomenon has more recently been described as the result of a social desirability bias. Thus a cultural awareness of how individuals perceive questions about their sexual histories may lead to a modification of the methods that clinicians use to gather this information. For example, if face-to-face interactions produce information of little validity then, perhaps, written or computer based questionnaires could be employed. In some situations, such approaches have already demonstrated their usefulness in gathering more accurate information.[54-56]

The structure of the PHAC questions gives the illusion that simple questions induce simple answers; an STI history, however, is anything but straightforward.[47] Many individuals do not know the signs and symptoms of STIs, and are, therefore, unable to report them with any degree of accuracy. [47] Further research has suggested that poor recognition of STI manifestations is context dependent. It seems to be dependent on a cultural sexual script regarding sexual education wherein cultural norms prohibit such knowledge.[57,58] For example, some studies have found that women from certain sub-Saharan African cultures are less knowledgeable about STIs and HIV, and are often unable to correctly identify personal symptoms. [59] Here, sexual script theory would help to inform clinical practice: while STI-like signs and symptoms (whether past or present) are strong indicators that an individual is infected with an STI, cultural norms (not necessarily patient recall) ultimately influence the nature and accuracy of the answers to questions about them. As pointed out above, the members of cultures which do not endorse sexual health education are less likely to be cognizant of STI symptoms.

The outcome of the foregoing is that the PHAC guidelines ignore how an individual's sexual practices, his/her willingness to discuss such information, and his/her ability to recognize signs of infection are all highly dependent on culture. This signifies that the findings of a sexual history assessment may not be accurate or representative. Sexual script theory emphasizes the importance of a multi-part analysis of the cultural, interpersonal, and intrapsychic aspects of human sexuality. Failure to do so results in clinicians gathering information without any understanding of the context (both globally and specifically) from which this information arises, thus undermining its usefulness because without such understanding, any assessment remains incomplete. 


\section{Clinical implications}

The above analysis reveals that while the PHAC guidelines (particularly related to relationship status, risk behaviour, and STI history) may be used to gather useful information about specific patients, these questions nevertheless ignore the significant influence that culture and dyadic (interpersonal) relations exert over human sexuality. Stated another way, the PHAC guidelines examine the act, the scene, the actor(s), and the performance - four of the five parts of Gagnon and Simon's[9] dramatic metaphor - however, the motivation (purpose, significance, and meaning) of specific sexual practices is not explored. The PHAC does not make any recommendations for exploring why patients have sex the way they do, why they have sex or meet sexual partners in the places they do, or why they have sex with the people they do. It is important to remember that a sexual health clinical interaction is not just about evaluating if an infection has already been acquired, but is also about (1) preventing future infections and (2) decreasing transmission by patients who are infected by incurable STIs or HIV.[47]

However, the foregoing problems with the PHAC STI/HIV assessment guidelines do not indicate that such assessments should not be undertaken. One might conclude, instead, that it could be beneficial if sexual health interviews were enhanced with other questions that explore the meaning and significance of different sexual practices for each patient. For example, clinicians might explore if patients have any questions or concerns about their sexuality (e.g., erectile dysfunction with condoms that results in decreased condom use), and could then advise these patients about how to indulge in their desires in ways that mitigate the likelihood of encountering or experiencing what the patients identify as unwanted/undesirable outcomes (e.g., STI/HIV acquisition, drug overdose, sexual assault, etc.). This constitutes an ideal opportunity for clinicians to use their expert knowledge about physiology, infectious disease and its transmission, pharmacology, and health care and prevention technologies to optimise their patients' health. Such an approach should be considered as quite distinct from the approach of instructing all patients to always use condoms.

The above discussion also suggests that questions regarding a patient's relationship status should not be eliminated; rather, they should be changed. Clinical assessments about relationships are often problematic because they are based on only one individual's account of the situation. This can present difficulties if they fail to acknowledge that each person's specific meaning and definition of the term, relationship, varies based on his/her cultural, interpersonal, and intrapsychic evaluations of sexual scripts. As such, because the PHAC guidelines fail to consider the cultural aspects of human sexuality, clinicians who follow these guidelines could inadvertently damage the therapeutic relationship that is needed in order to gather highly sensitive information about patients' sexual practices. In fact, clinicians may unknowingly disregard concerns that their patients' feel are legitimate, while conversely, patients' may overlook their clinicians counselling because they feel that the concern that the clinician is addressing is not actually a problem. In these cases, the lack of direction in the PHAC guidelines about the need to appreciate the cultural level of human sexuality could severely weaken the effectiveness of these guidelines during a clinical interaction.

As a caveat, however, the discussion presented above does not suggest that clinicians should absolutely forego any form of assessment of their patient's sexual behaviour; to do so would be to overlook the information that guides much clinical work. However, if clinicians ignore the significant impact of interpersonal and cultural level influences on sexual behaviour, they will miss important aspects that greatly impact on human sexual behaviour. In order to avoid alienating their patients, clinicians must be sensitive to their real-life situations: they must be able to comprehend how the harm reduction strategies that they suggest may, or may not, be feasible. Some patients, particularly women, may be powerless to suggest condom use due to the prevailing cultural norms that structure their social interactions. As such, clinical guidance that is unrealistic on an interpersonal level is ultimately useless information. Fears of ostracism, violence, or exclusion may overpower intrapsychic beliefs about the health benefits of using condoms. In other words, clinicians must move beyond their own intrapsychic interpretations of their personal sexual scripts when they are engaging in sexual health practice.

\section{Conclusion}

According to Gagnon and Simon, [9] science is a product of the culture and time period in which it exists and, therefore, cannot be completely objective. When this idea is extended to the 2006 PHAC guidelines, it means that it is impossible for them to be completely objective. Instead, they are a product of the health care culture in which they were created, and are thus a component of that sexual script. When using the PHAC[8] guidelines to assess sexual behaviours in individuals, it quickly becomes apparent that the individual is reduced to his or her potential for acquiring and transmitting 
STIs and HIV. The guidelines do not acknowledge individuals in the context of their socio-political cultures and history. The sexual scripts of individuals[9] are mostly ignored in favour of questions that encourage quantitative answers instead of qualitative knowledge. Clearly, the PHAC[8] guidelines do not form a comprehensive STI/HIV assessment because, unfortunately, a failure to incorporate the cultural and interpersonal aspects of human sexual interactions will result in a very shallow sexual health assessment for any individual patient. In light of Gagnon and Simon's sexual script theory, it is evident that additions to the PHAC guidelines must be made so that clinicians can ascertain more comprehensive pictures of their patients. In this way, realistic clinical advice that is more easily and willingly followed by our clients could be developed.

\section{References}

1.PHAC (Public Health Agency of Canada). Reported cases of notifiable STI from January 1 to June 30, 2008 and January 1 to June 30, 2009 and corresponding annual rates for the years 2008 and 2009 [document on the internet]. 2010 [cited February 12th, 2010]. Available from http://www.phac-aspc. gc.ca/std-mts/stdcases-casmts/cases-cas-09-eng.php.

2.PHAC (Public Health Agency of Canada). HIV and AIDS in Canada: Surveillance Report to December 31, 2008 [document on the internet]. 2009. [cited February 1st, 2010]. Available from: http://www.phac-aspc.gc.ca/aids-sida/ publication/index-eng.php\#surveillance.

3.Aral SV, Lipshutz JA, Douglas JM. Introduction. In: Aral AO, Douglas JM, Lipshutz JA, editors. Behavioural Interventions for Prevention and Control of Sexually Transmitted Diseases. New York: Springer, 2007; ix-xix.

4.Lawrence JS, Fortenberry JD. Behavioural interventions for STDs: theoretical models and intervention methods. In: Aral SO et al., editors. Behavioural Interventions for Prevention and Control of Sexually Transmitted Diseases. New York: Springer Science, 2007;23-59.

5.Leviton LC. Integrating psychology and public health: challenges and opportunities. American Psychologist 1996; 51:42-51.

6. Manhard L, Holmes KK. Randomized controlled trials of individual-level, population-level, and multilevel interventions for preventing sexually transmitted infections: what has worked? Journal of Infectious Diseases 2005; 191:S7-24.

7.Nation M, Crusto C, Wandersman A, et al. What works in prevention: principles of effective prevention programs. American Psychologists 2003; 56:449-56.

8.PHAC (Public Health Agency of Canada). Canadian Guidelines on Sexually Transmitted Infections, 2006 Ed. Ottawa: Queen's Printer; 2006.

9.Gagnon JH, Simon W. Sexual conduct: The social sources of human sexuality. Chicago: Aldine, 1973.

10.Gagnon JH, Simon W. Sexual conduct: The social sources of human sexuality. 2nd ed. Chicago: Aldine, 2005.

11. Langdridge D, BarkerM, editors. Situating Sadomasochism. In: Safe, Sane, and Consensual: Contemporary Perspectives on Sadomasochism. Basingstoke: Palgrave Macmillian, 2007; 3-9.

12. Kleinplatz PJ, Moser C. Is SM Pathological? In: Langdridge D, Barker M, editors. Safe, Sane, and Consensual: Contemporary Perspectives on Sadomasochism. Basingstoke: Palgrave Macmillian, 2007; 55-62.

13.MacKendrick, K. Counterpleasures. New York: State University of New York Press, Albany; 1999.

14.Butler J. Gender Trouble: Feminism and the Subversion of Identity. New York: Routledge, 1999.

15.Goffman E. The presentation of self in everyday life: Selections. In: Newman DM, O'Brien JA, editors. Sociology: Exploring the Architecture of Everyday Life. Thousand Oaks: Pine Forge Press, 2008; 120-8.

16. Simon W, Gagnon JH. A sexual scripts approach. In: Geer $\mathrm{JH}, \mathrm{O}$ 'Donohue, editors. Theories of human sexuality. New York: Plenum, 1987; 363-83.

17. Gagnon JH. An interpretation of desire: Essays in the study of sexuality. Chicago: University of Chicago Press, 2004.

18.Foucault M. The Use of Pleasure: The History of Sexuality. Vol 2. New York: Vintage, 1985.

19.Foucault M. The Care of the Self: The History of Sexuality. Vol 3. New York: Vintage, 1986.

20.Simon W, Gagnon JH. Sexual scripts: Permanence and change. Archives of sexual behavior 1986; 13:97-120.

21.Gagnon JH. Scripts and the coordination of sexual conduct. In: Cole JK, Dienstbier R, editors. Proceedings of the Nebraska symposium on motivation. Lincoln: University of Nebraska Press 1974; 27-59.

22.Gagnon JH. Human sexualities. Glenview (IL): Scott Foresman; 1977 
23. Rubin G. The traffic in women. In: Reiter RR, editor. Toward an anthropology of women. New York: Monthly Review; 1975.

24.Rubin G. Thinking sex. In: Vance C, editor. Pleasure and danger. London: Routledge: Kegan Paul; 1984.

25.Sisson K. The cultural formation of S/M: history and analysis. Lesbian \& Gay Psychology Review 2005;6(3):14762.

26. Humphreys L. Tearoom Trade: Impersonal Sex in Public Places. New Jersey: Transaction, 1970.

27. Holmes D, Gastaldo D, O'Byrne P, Lombardo A. Bareback Sex: A Conflation of Risk and Masculinity. International Journal of Men's Health 2008; 7(2):171-91.

28. Holmes D, O'Byrne P, Gastaldo D. Setting the Space for Sex: Architecture, Desire and Health Issues in Gay Bathhouses. International Journal of Nursing Studies 2007; 44:273-84.

29. Holmes D, O'Byrne P, Gastaldo D. Raw Pleasure as Limit Experience: A Foucauldian Analysis of Unsafe Anal Sex between Men. Social Theory \& Health 2006; 4:319-33.

30. Holmes D, O'Byrne P. The Art of Public Health Nursing: Using Confession Technè in the Sexual Health Domain. Journal of Advanced Nursing 2006; 56(4):1-8.

31.Goffman E. The Presentation of Self in Everyday Life. New York: Anchor Books, 1959.

32.Fehr E. Third-party punishment and social norms. Evolution and Human Behaviour 2004; 25:63-87.

33. Gorbach PM, Holmes KK. Sexual partnership effects on STIs/HIV transmission. In: Holmes KK et al., editors. Sexually Transmitted Diseases. New York: McGraw Hill, 2008;12736.

34.Gorbach PM, Drumright LN, Holmes KK. Discord, discordance and concurrency: comparing individual and partnership-level analyses of new partnerships of young adults at risk of STIs. Sexually Transmitted Diseases 2005; 31(1):7-12.

35.Ellish NJ, Celentano D, Zenilman JM. Reliability of partner reports of sexual history in a heterosexual population at a sexually transmitted diseases clinic. Sexually Transmitted Diseases 1996; 23:528-42.

36.Gupta GR. How men's power over women fuels the HIV epidemic. British Medical Journal 2002; 324:183-4.

37.Guzman R, Colfax G, Wheeler S, et al. Negotiated safety relationship and sexual behaviour among a diverse sample of HIV-negative men who have sex with men. Journal of Acquired Immune Deficiency Syndrome 2005; 38(1):82-6.

38.Elford JBG, MacGuire M, Sherr L. Gay men, risk and relationships AIDS 2001; 15(8):1053-5.

39.Sharpe PA, Brandt HM, McCree DH. Knowledge and beliefs about abnormal Pap test results and HPV among women with high-risk HPV: results from in-depth interviews. Women \& Health 2005; 42:107-33.

40. Holmes D, Warner D. The anatomy of a forbidden desire: men, penetration and semen exchange. Nursing Inquiry 2005; 12:10-20.

41.O'Byrne P. Eroticizing the abject: Understanding the role of skeeting in sexual practices. In: Holmes D, Rudge T, editors. Abjectly Boundless. London: Ashgate Press, 2010; 81-99.

42. Marston C, King E. Factors that shape young people's sexual behaviour: a systematic review. The Lancet 2006; 368(9547):1581-6.

43.McCree DH, Eke A, Williams SP. Dyadic, Small group, and community-level behavioural interventions for STD/HIV prevention. In: Aral SO et al., eds. Behavioural Interventions for Prevention and Control of Sexually Transmitted Diseases. New York: Springer Science, 2007;105-24.

44.O'Byrne P, Holmes D. The Microfascism of the Social Contract: Reframing our Understanding Public Health STI Control. Surveillance Society 2009; 7(1):1-13.

45.O'Byrne P. The Dissection of Risk: A Conceptual Analysis. Nursing Inquiry 2008; 15(1):30-9.

46.O'Byrne P, Holmes D. The Micro-Fascism of Plato's Good Citizen: Producing (Dis)order through the Construction of Risk. Nursing Philosophy 2007; 8:93-100.

47.Kurth AE, Speilberg F. Individual-level risk assessment for sexually transmitted infections, including HIV. In: Holmes KK et al., editors. Sexually Transmitted Diseases. New York: McGraw Hill, 2008; 877-901.

48.Bird J, Cohen-Cole SA. The three-function model of the medical interview: an education device. Advances in Psychosomatic Medicine 1990; 20:65-88.

49.Garnett GP. The transmission dynamics of sexually transmitted infections. In: Holmes KK et al., editors. Sexually Transmitted Diseases. New York:McGraw Hill, 2008; 27-39.

50.Morris M, Goodreau S, Moody J. Sexual networks, concurrency, and STD/HIV. In: Holmes KK et al., editors. 
Sexually Transmitted Diseases. New York: McGraw Hill, 2008; 109-25.

51.Ndulo J, Faxelid E, Tishelman C, Krantz I. "Shopping" for sexually transmitted disease treatment: focus group discussions among lay persons in rural and urban Sambia. Sexually Transmitted Diseases 2000; 27(9):496-503.

52.Scoular A, Duncan B, Hart G. "That sort of place ... where filthy men go": a qualitative study of women's perceptions of genitourinary medicine services. Sexually Transmitted Infection 2001; 77:340-43.

53.Fortenberry JD, Dennis J, McFarlane M, et al. Relationship of stigma and shame to gonorrhea and HIV screening. American Journal of Public Health 2002; 92(3):378-81.

54. Hasley S. A comparison of computer-based and personal interviews for the gynaecological history update. Obsetrics and Gynecology1995; 85:494-8.

55.Kurth AE, Martin D, Golden $M$, et al. A comparison between audio computer-assisted self-interviews and clinician interviews for obtaining the sexual history. Sexually Transmitted Diseases 2004; 31(12):719-26.

56.Ghanem KG, Hutton HE, Zenilman JM, Zimba R, Erbelding EJ. Audio computer assisted self interview and fact to face interview modes in assessing response bias among STD clinic patients. Sexually Transmitted Infections 2005; 81(5):421-5.

57.Voeten H, O’Hara H, Kusimba J, et al. Gender differences in health care-seeking behaviour for sexually transmitted diseases. Sexually Transmitted Diseases 2004; 31(5):265-72.

58.Go CF, Quan VM, Chung A, Zenilman J, Hanh VTM, Celentano D. Gender gaps, gender traps: sexual identity and vulnerability to sexually transmitted diseases among women in Vietnam. Social Science \& Medicine 2002;55(3):467-81.

59. Hawken MP, Melis R, Ngombo D, et al. Opportunity for prevention of HIV and sexually transmitted infections in Kenyan youth: results of a population-based survey. Journal of AIDS 2002;31:529-35.
Contact Information for Author:

Patrick O'Byrne, RN, Ph.D.

Assistant Professor

University of Ottawa

Faculty of Health Sciences

School of Nursing

451 Smyth Road

Ottawa, Ontario, K1H 8 M5

Canada

Email: pjobyrne@uottawa.ca

Jessica Watts, RN, B.Sc.N.

Master's Student and Research Assistant

University of Ottawa

Faculty of Health Sciences

School of Nursing 